\title{
Nonlinear Time-Series Analysis of Pulsation of Post-AGB Stars by Genetic Algorithm/Neural Network Hybrid Systems
}

\author{
Toshiki Aikawa \\ Tohoku Gakuin University, Izumi-ku, Sendai 981-3193, Japan
}

\begin{abstract}
Some pulsating post-AGB stars have been observed with an Automatic Photometry Telescope (APT) and a considerable amount of precise photometric data has been accumulated for these stars. The datasets, however, are still sparse, and this is a problem for applying nonlinear time series: for instance, modeling of attractors by the artificial neural networks (NN) to the datasets. We propose the optimization of data interpolations with the genetic algorithm (GA) and the hybrid system combined with NN. We apply this system to the Mackey-Glass equation, and attempt an analysis of the photometric data of post-AGB variables.
\end{abstract}

\section{Introduction}

The artificial neural networks, especially recurrent type networks, have been successfully applied to analyses of time-series data even for chaotic data. Some variable star's also show chaotic behavior and nonlinear time series analysis, for instance recurrent networks, are desirable for studying these stars. We must, however, prepare successively sampled data. In photometric observations for variable stars there are many reasons for interruptions in ground-based observations. Thus, we must fill time-series data with missing data points. In this report, we propose the optimization of the data interpolations for the missing data by using the genetic algorithms along with the neural networks for finding attractors in the time-series data.

\section{Hybrid Systems of GA/NN}

Genetic Algorithm-The value for interpolation at a data point when there is no actual observed data is assigned as an 8 bits binary string in a long artificial chromosome. The chromosome consists of the array of the 8 bits binary string. The size of the array is equal to the total number of the datapoints to be interpolated. We computationally perform evolution of a population in which each individual has the same structure of chromosome, but different values of the binary string, so different interpolated values for the same missing point.

Recurrent Neural Network-We use a three-layered feed-forward network. The input layer has 4 neurons, and the output layer has 1 neuron. For network training, we prepare a set of data which consists of four successive datapoints in 
the original time series for input, and one data point at the next datapoint in the original time series for output. A sequence of the set is prepared for all of the data in the time series. The fitness of genetic individual of the population is evaluated by the degree of the convergence of the neural network checked after a certain amount of training. We confirm a very tight correlation between the convergence degree of the NN and the mean deviation of the interpolated values from the true ones.

\section{Mackey-Glass Equations}

The time-series data obtained from the following delayed differential equation (Mackey-Glass equation) are analyzed:

$$
\frac{d x}{d t}=\frac{a x(t-\tau)}{1+[x(t-\tau)]^{10}}-b x(t)
$$

where $a=1 / 5$ and $b=1 / 10$. For the delay parameter $\tau=17$ and 30 , with the sampling time-interval $\Delta=6$, the equation yields chaotic time series with the fractal dimension, 2.1 and 3.6 , respectively.

We prepare 512 points, but we assume that some of them are missing datapoints and so the data values should be obtained by interpolation. We performed the simulation with 400 populations up to 400 generation steps. We confirm that GA can do reasonably good interpolations for missing datapoints and NN can get attractors quite similar to original ones, even for cases where the total missing data is more than $30 \%$.

\section{Post-AGB Stars}

There are F-type supergiant stars which show small amplitude variability. Some of them show infrared excess and are believed in the post-ABG stages in their evolution. The variability of these stars has been observed for a long span using the ATP facility for $89 \mathrm{Her}$ and HD 161796. The photometric data on these stars available from the web site http://ddo.astro.utoronto.ca/photometry have been used. We arrange the data for one point for each day at UT $=0$. For $89 \mathrm{Her}$, the span of the whole dataset is $2995 \mathrm{~d}$, and we have 725 points actually observed; for HD 161796 , the span is $2678 \mathrm{~d}$, and 650 points for actual observed data. Initial values of photometric values at the missing days are random numbers within the range of the observed variability. We perform the simulation with 400 populations up to 400 generations for both the variables. So far we have not succeeded in getting good convergence.

\section{Conclusions}

As shown in the case of Mackey-Glass equation, this technique is applicable to time-series data which have interruptions with degree of a few $10 \%$. But severer constraints, more than the range of the variability for the interpolations at missing datapoints, should be necessary for practical use for photometric observations. 